\title{
Design of a nonscaling fixed field alternating gradient accelerator
}

\author{
D. Trbojevic, ${ }^{*}$ E. D. Courant, and M. Blaskiewicz \\ BNL, Upton, New York 11973, USA \\ (Received 7 February 2005; published 19 May 2005)
}

\begin{abstract}
We present a design of nonscaling fixed field alternating gradient accelerators (FFAG) minimizing the dispersion action function $H$. The design is considered both analytically and via computer modeling. We present the basic principles of a nonscaling FFAG lattice and discuss optimization strategies so that one can accelerate over a broad range of momentum with reasonable apertures. Acceleration schemes for muons are discussed.
\end{abstract}

DOI: 10.1103/PhysRevSTAB.8.050101

PACS numbers: $29.20 .-\mathrm{c}, 41.75 . \mathrm{Lx}$

\section{INTRODUCTION}

The fixed field alternating gradient (FFAG) configuration, introduced independently by Ohkawa [1], Symon [2], and Kolomensky [3], has received much attention in recent years. A "proof of principle" machine has been built at KEK [4], followed by a $150 \mathrm{MeV}$ proton synchrotron which is being commissioned [5]. In the scaling FFAG design the particle orbits "scale" with momentum, and acceleration over a large range of momentum requires large apertures. In nonscaling FFAGs the aperture requirements can be significantly reduced. ${ }^{1}$ Nonscaling FFAGs have been discussed as a part of the general FFAG family [8] and in the context of muon acceleration [9-14], where the short muon lifetime prohibits slow ramping of the magnetic fields. The FFAG acts similar to a recirculating linear accelerator (RLA), but all the orbits go through the same lattice, obviating the need for separated arcs.

\section{A. The Basic Cell}

We end up with the rather simple configuration of Fig. 1. The accelerator is composed of a large number (66 in our case) of identical unit cells. Each cell contains a magnet triplet, with a relatively long gradient bending magnet $Q D$ ("combined function") having a strong central field and negative gradient (horizontally defocusing) at the center, flanked by a pair of negative bend magnets $Q F$ that are horizontally focusing, and then a drift space about $2 \mathrm{~m}$ long to accommodate an accelerating cavity.

The cell is symmetric with respect to the center of the defocusing combined function dipole and the center of the long drift. The horizontal and vertical betatron functions $\beta_{x}$ and $\beta_{y}$ and the dispersion function in the basic cell, at the reference momentum, are shown in Fig. 2.

\footnotetext{
*Electronic address: dejan@bnl.gov

${ }^{1}$ In the recent $10-20 \mathrm{GeV}$ scaling FFAG lattice for muon acceleration in KEK Japan the orbit offsets were reported [6] to be of the order of $40 \mathrm{~cm}$. A design of the nonscaling FFAG with the same circumference [7] produces maximum orbit offsets between $\pm 42 \mathrm{~mm}$.
}

\section{PARTICLE MOTION AT VERY LARGE MOMENTUM OFFSET}

We consider a particle of momentum $p$ and a reference particle with momentum $p_{0}$ and charge $q$; the momentum offset is $\delta=\left(p-p_{0}\right) / p_{0}$. The magnetic rigidity of the reference particle is $(B \rho)_{0}=p_{0} / q$; the reference particle is on a reference orbit (assumed planar) with local radius of curvature $\rho_{0}$ and vertical field $B_{0}(s)=(B \rho)_{0} / \rho_{0}(s)$. In the cases considered here the field $B_{0}(s)$ and with it the radius of curvature $\rho_{0}(s)$ are constant in each magnet, so that the reference orbit consists of circular arcs in the magnets and straight sections between the magnets; we also assume that the magnet edges are straight at right angles to the reference orbit. We assume that the magnetic field in the dipole magnets is linear:

$$
B_{y}=B_{0}+G x,
$$

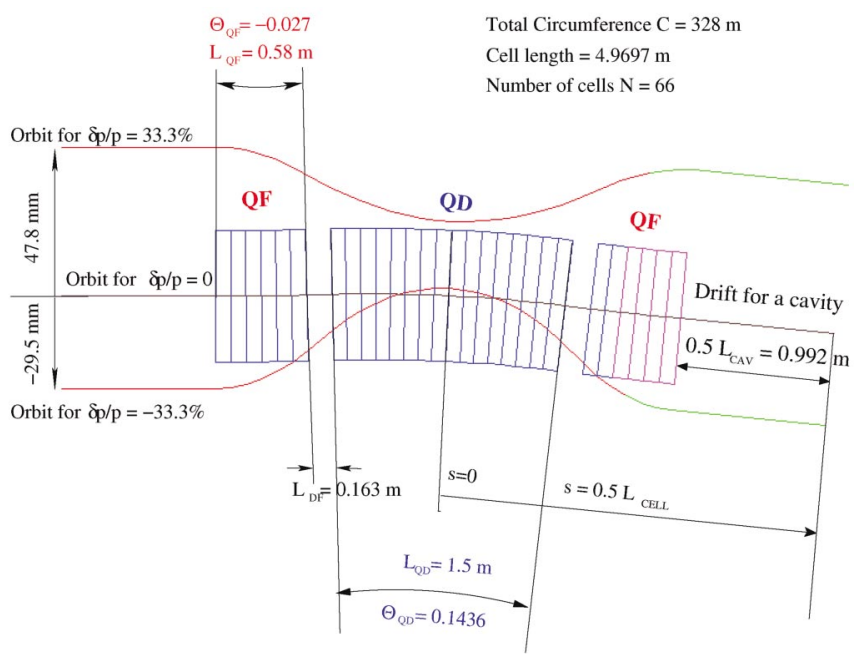

FIG. 1. (Color) Basic half cell. The cell is symmetric with respect to $s=0$. The total length of the cell equals $L_{\text {cell }}=$ $L_{\text {cavity }}+2 * L_{Q F}+L_{Q D}+2 * L_{D F}$. There are two types of magnets, $Q D$ (normal bend, field $B_{Q D}=4.95 \mathrm{~T}$ ) and $Q F$ (negative bend, field $B_{Q F}=-2.29 \mathrm{~T}$ ). Each has constant gradient, $G_{Q F}=57.19 \mathrm{~T} / \mathrm{m}$ and $G_{Q D}=-34.27 \mathrm{~T} / \mathrm{m}$. 


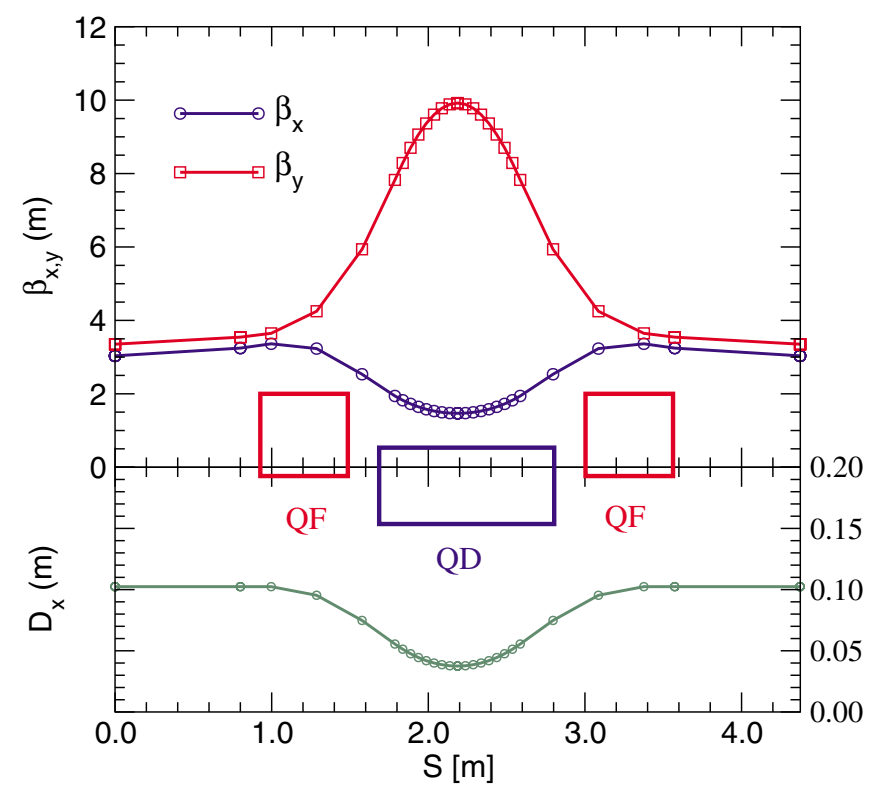

FIG. 2. (Color) Betatron functions and dispersion in the basic cell at the central energy.

$$
B_{x}=G y \text {, }
$$

with no higher order terms in $x$ and $y$. Here $x$ and $y$ are the horizontal and vertical deviations from the reference orbit; the central field $B_{0}$ is positive in $Q D$ and negative in $Q F$ while the gradient $G$ is positive in $Q F$ and negative in $Q D$.

For muon acceleration we have to accelerate from 10 to $20 \mathrm{GeV}$ very quickly because of the short decay time of the muon. Suitable superconducting cavities for muon acceleration have been developed at Cornell University [15]; these require at least two meters of drift space. Therefore we have to provide this much space in each cell. The lengths, fields, and gradients of the magnets are adjusted so that the total bend angle of the reference orbit through one cell is

$$
\frac{2 \pi}{N_{\text {cell }}}=\frac{L_{Q D}}{\rho_{Q D}}-\frac{2 L_{Q F}}{\rho_{Q F}},
$$

and the horizontal and vertical betatron oscillations are stable for all energies from 10 to $20 \mathrm{GeV}$, i.e., $\delta$ ranging from -0.33 to 0.33 with the central momentum at $p_{0}=$ $15 \mathrm{GeV} / c$. Because of the high acceleration rate it is permissible for the betatron tunes to vary over the energy range as long as they stay between about 0.05 and 0.45 per period.

\section{A. Accuracy of accelerator physics codes}

The design of a fixed field accelerator requires design codes accurate for large momentum ranges. We have previously reported [16] a study of several accelerator physics lattice design codes for a large momentum range. The tunes and the Courant-Snyder amplitude functions were calculated by several different codes and compared to the

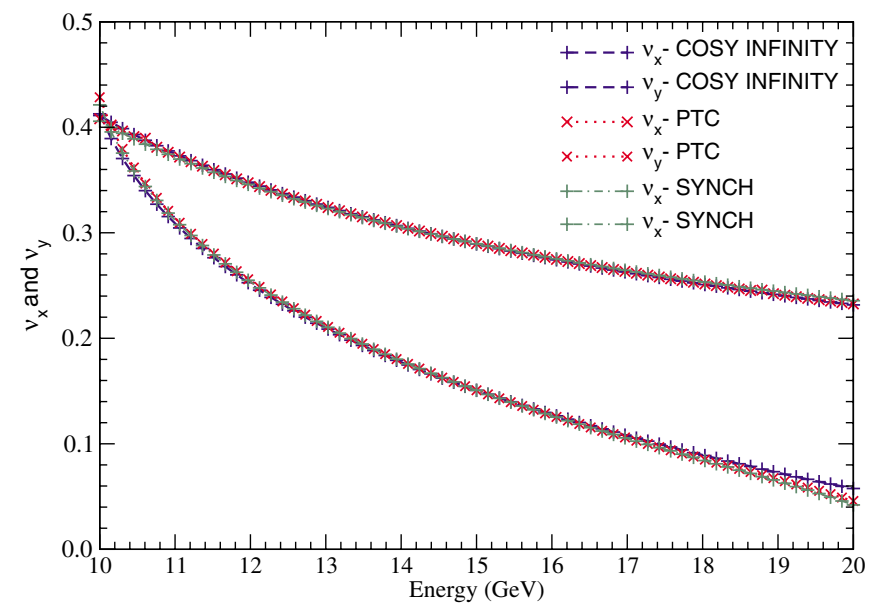

FIG. 3. (Color) Betatron tunes $\nu_{x}$ and $\nu_{y}$ calculated for the lattice of Fig. 1 by the codes PTC, COSY INFINITY, and SYNCH over the muon acceleration range from 10 to $20 \mathrm{GeV}$.

analytical predictions for the case of a simple test lattice consisting of five identical $72^{\circ}$ dipoles, with fixed linear fields. In addition, the FFAG examples designed using the program SYNCH [17] were cross checked by Forest with the code PTC [18], by Berg using the COSY INFINITY code [19] up to 8th order in $\delta$, and by Méot with the ZGOUBI-Saclay code [20]. Results from the "ZGOUBI" test were shown at the KEK FFAG workshop [21]. A comparison between calculations using the codes PTC, COSY INFINITY, and $\mathrm{SYNCH}$, in our FFAG lattice for muon acceleration over the whole momentum range of the tunes is presented in Fig. 3, and of the maximum of the radial orbit offsets in Fig. 4. A comparison of the path length calculations by the three codes is shown in Fig. 5.

The exact equations of the particle motion in a nonscaling FFAG for very large momentum offsets have been previously discussed in detail [9,22,23,25-27].

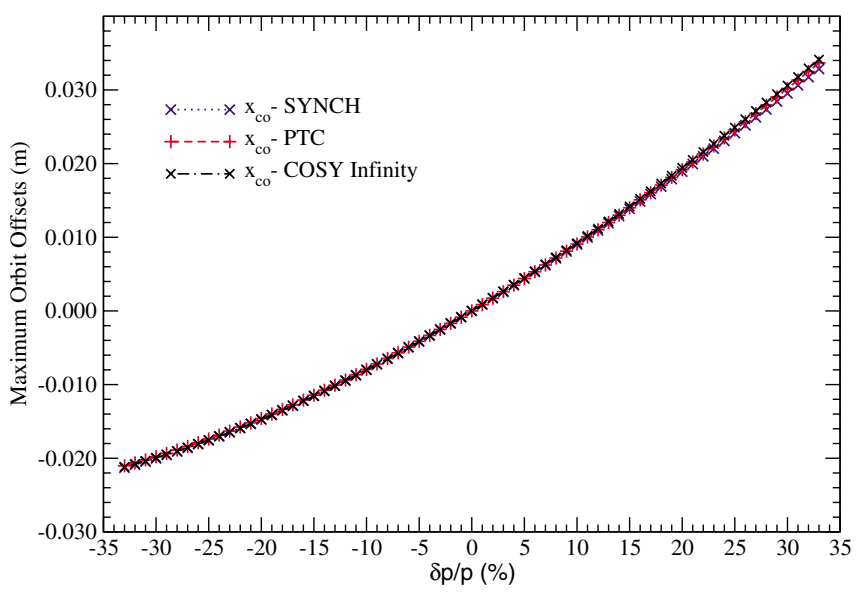

FIG. 4. (Color) Comparison of the maximum orbit offset calculated by the three codes: PTC, COSY INFINITY, and SYNCH, over the muon acceleration range from 10 and $20 \mathrm{GeV}$. 


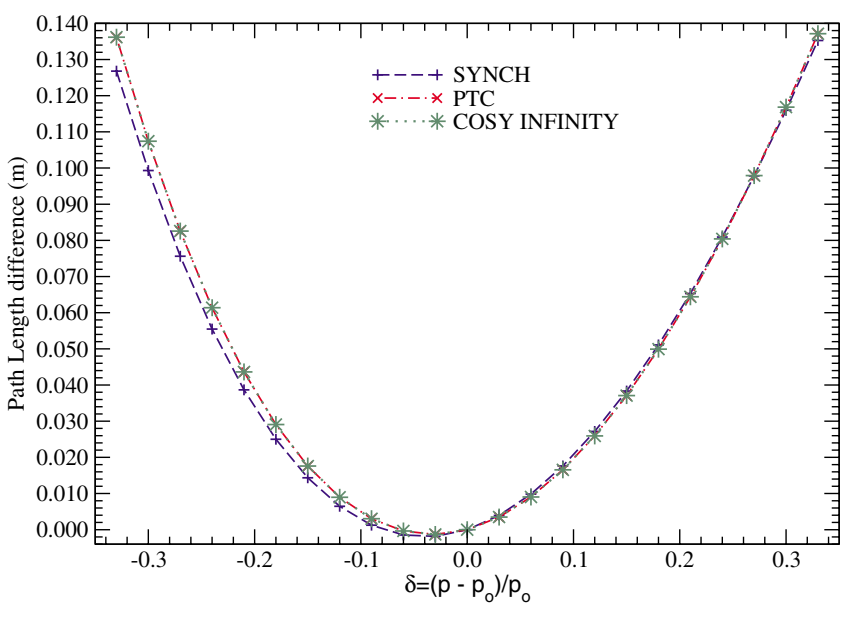

FIG. 5. (Color) Comparison of the path length calculations by the three codes SYNCH, PTC, and COSY INFINITY over the muon acceleration range from 10 and $20 \mathrm{GeV}$. Results obtained by the PTC and COSY INFINITY codes are almost identical.

\section{CONTROL OF THE AMPLITUDE OF THE DISPERSION FUNCTION}

The dependence of radial offset on momentum is given by $\Delta x=D_{s} \delta$, where $D_{s}$ is the horizontal dispersion function. A small dispersion function makes for large momentum acceptance. If the aperture is to be less than $4 \mathrm{~cm}$ the dispersion function has to be $D_{s}<12 \mathrm{~cm}$ in the whole momentum range.

A useful measure of the variation of path length is the dispersion action function [28] (the Courant-Snyder invariant of the dispersion function $D$ ), defined as

$$
H\left(D, D^{\prime}\right)=\left(\frac{D}{\sqrt{\beta}}\right)^{2}+\left(D^{\prime} \sqrt{\beta}+\frac{\alpha D}{\sqrt{\beta}}\right)^{2} .
$$

The normalized dispersion coordinates are

$$
\begin{gathered}
\xi=\frac{D}{\sqrt{\beta}} \quad \text { and } \quad \chi=D^{\prime} \sqrt{\beta}+\frac{\alpha D}{\sqrt{\beta}} ; \\
H\left(D, D^{\prime}\right)=\xi^{2}+\chi^{2} .
\end{gathered}
$$

To minimize aperture it is advantageous to minimize the function $H$. It suffices to minimize the average function $\langle H\rangle$ within the main bending element. This could be easily understood by observing that the maximum of the vector $\sqrt{H}_{\max }$ is at the end of the major bend, as shown in the normalized dispersion plot in Fig. 6. Figure 6 shows the

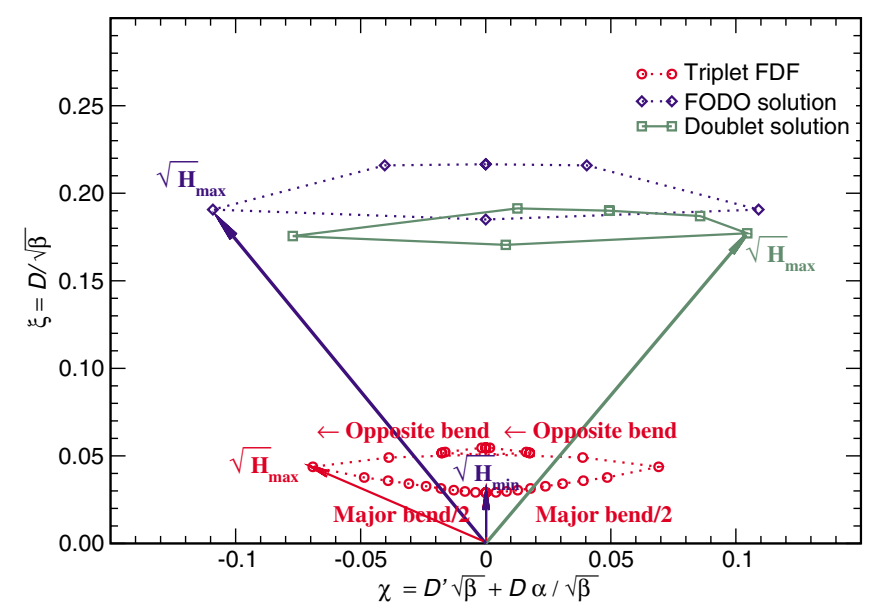

FIG. 6. (Color) Normalized dispersion function in the basic cell for three designs: FODO cells, doublets, and our triplets using the same magnets.

normalized dispersion function within the basic triplet cell, as well as that for the focusing-drift-defocusing-drift (FODO) and doublet cells (FD) designed with the same magnets. The magnet lengths are $L_{Q F}=0.58 \mathrm{~m}$ and $L_{Q D}=0.85 \mathrm{~m}$. Other details for these three different cells are shown is the Table I.

The advantage of our triplet cell compared to the doublet or FODO structures is explained by the significantly smaller values of $\sqrt{H}_{\max }$.

Muon acceleration is performed near the top of the sinusoidal rf wave, with a small number of turns and a large energy gain per turn. As discussed in the section on acceleration (see below) this necessitates a fixed rf frequency. Therefore it is desirable that the time of travel around the circumference vary as little as possible with momentum. Since the muons are relativistic this is equivalent to having the orbit length vary as little as possible with $\delta$. is

The variation in the particle path length around the ring

$$
\begin{aligned}
\Delta C & =\oint_{C}\left(\sqrt{\left(1+\frac{x}{\rho}\right)^{2}+x^{\prime 2}+y^{\prime 2}}-1\right) d s \\
& \cong\left[\oint \frac{D(s)}{\rho} d s\right] \delta .
\end{aligned}
$$

The lattice with the smallest dispersion function gives both

TABLE I. A comparison between designs by the FODO, DOUBLET, or TRIPLET cells. The cells properties include the length of the cells, gradient and magnet strengths, and bending angles

\begin{tabular}{lccccccc}
\hline \hline TYPE & $L_{\text {cell }}(\mathrm{m})$ & $G_{Q F}(\mathrm{~T} / \mathrm{m})$ & $G_{Q D}(\mathrm{~T} / \mathrm{m})$ & $\theta_{Q F}(\mathrm{rad})$ & $\theta_{Q D}(\mathrm{rad})$ & $B_{Q F}(\mathrm{~T})$ & $B_{Q D}(\mathrm{~T})$ \\
\hline FODO & 2.2237 & 64.792 & -68.214 & -0.02087 & 0.12440 & -1.80 & 7.323 \\
DOUBLET & 2.2162 & 65.258 & -68.914 & -0.02190 & 0.12873 & -1.89 & 7.577 \\
TRIPLET & 2.2162 & 66.464 & -70.112 & -0.02179 & 0.12850 & -1.88 & 7.564 \\
\hline \hline
\end{tabular}


the smallest orbit offsets and the shortest path length differences over the range of momenta. The average value of the dispersion action in the central dipole and the conditions for its minimum are given by the equations

$$
\langle H\rangle=\frac{1}{L} \int_{0}^{L} H(s) d s, \quad \frac{\partial\langle H\rangle}{\partial D_{0}}=0, \quad \frac{\partial\langle H\rangle}{\partial \beta_{0}}=0 .
$$

The dispersion function, its slope, and the Courant-Snyder functions in the central dipole are

$$
\begin{gathered}
D(s) \cong \frac{1}{\rho K}(\cosh \phi-1)+D_{0} \cosh \phi \\
=\left(D_{0}+\frac{1}{\rho K}\right) \cosh \phi-\frac{1}{\rho K}, \\
D^{\prime}(s) \cong\left(D_{0} \sqrt{K}+\frac{1}{\rho \sqrt{K}}\right) \sinh \phi+D_{0}^{\prime} \cosh \phi \\
=\sqrt{K}\left(D_{0}+\frac{1}{\rho K}\right) \sinh \phi, \\
\beta(s) \cong\left(\beta_{0}+\frac{\gamma_{0}}{K}\right) \cosh \phi-\frac{\gamma_{0}}{K} \cosh \phi-\frac{\gamma_{0}}{K}, \\
\alpha(s) \cong-\sqrt{K}\left(\beta_{0}+\frac{\gamma_{0}}{K}\right) \sinh \phi \cosh \phi, \\
\gamma(s) \cong K\left(\beta_{0}+\frac{\gamma_{0}}{K}\right) \cosh ^{2} \phi-K \beta_{0},
\end{gathered}
$$

where the defocusing strength $K$ is

$$
K(s)=\frac{G}{B \rho}-\frac{1}{\rho^{2}(s)},
$$

and the betatron phase is $\phi=\sqrt{K} s$. The dispersion action function $H$ in the dipole is

$$
\begin{aligned}
H(s)= & \frac{1}{\rho^{2} K}\left(\beta_{0}+\frac{\gamma_{0}}{K}\right) \cosh ^{2} \phi-\frac{2 \gamma_{0}}{\rho K}\left(D_{0}+\frac{1}{\rho K}\right) \\
& \times \cosh (\phi)-\frac{\beta_{0}}{\rho^{2} K}+\gamma_{0}\left(D_{0}+\frac{1}{\rho K}\right)
\end{aligned}
$$

and its average value $\langle H\rangle$ is

$$
\begin{aligned}
\langle H\rangle= & \frac{2}{L} \int_{0}^{L / 2} H(s) d s, \\
\langle H\rangle= & \frac{\theta^{2}}{2 q^{2}}\left(\beta_{0}+\frac{L^{2}}{\beta_{0} q^{2}}\right)\left(\frac{\sinh q}{q}-1\right)-\frac{4 \theta L}{\beta_{0} q^{3}}\left(D_{0}+\frac{L \theta}{q^{2}}\right) \\
& \times \sinh \frac{q}{2}-\frac{\beta_{0} \theta^{2}}{q^{2}}+\frac{1}{\beta_{0}}\left(D_{0}+\frac{L \theta}{q^{2}}\right) .
\end{aligned}
$$

We minimize $\langle H\rangle$ with respect to the initial values of $\beta_{0}$ and $D_{0}$ at the center of the defocusing central bending element, by setting the partial derivatives $\partial\langle H\rangle / \partial D_{0}$ and $\partial\langle H\rangle / \partial \beta_{0}$, equal to 0 . The solutions are

$$
\begin{gathered}
D_{0}=\frac{2 L \theta}{q^{3}} \sinh \frac{q}{2}-\frac{L \theta}{q^{2}}, \\
\beta_{0}=\frac{L}{q} \sqrt{\frac{\frac{4}{q^{2}} \sinh ^{2} \frac{q}{2}-\frac{1}{2}\left(1+\frac{\sinh q}{q}\right)}{1-\frac{1}{2}\left(1+\frac{\sinh q}{q}\right)},}
\end{gathered}
$$

where $q=L \sqrt{K}$ and $\theta=L / \rho$ as previously defined [29]. With the magnet lengths and strengths as given in Fig. 1 we use Eqs. (16) and (17) to set the central values of $\beta_{0}$ and $D_{0}$ and, using the SYNCH code, solve for the focusing strengths and gap dimensions to obtain zero slope at the symmetry points. We then calculate the lattice functions for a range of momenta from $\delta=-0.33$ to $\delta=+0.33$. But we find that this lattice is unstable below $14.5 \mathrm{GeV}$ when the central energy is $15 \mathrm{GeV}$. Since we need stability in the whole range between 10 and $20 \mathrm{GeV}$, this lattice is clearly not acceptable. Therefore the next step is to search for stable solutions in a larger momentum range by setting larger initial values of $\beta_{x_{0}}$ and $D_{x_{0}}$. Unfortunately, with larger value of the initial $\beta_{x_{0}}$ and $D_{x_{0}}$ the maximum orbit offsets, maximum values of the $H$ function, dispersion, and variation of path length around the ring with momentum, also increase. The best solution with a stable lattice and the required range in muon energy from 10 to $20 \mathrm{GeV}$ is the one of Fig. 1.

\section{A. A search for a large acceleration range in $\delta p / p$}

A lattice cell is constructed at the central muon energy of $15 \mathrm{GeV}$ with the horizontal and vertical tunes to be in the middle of the range $0.1<\nu_{x}, \nu_{y}<0.5$. A search for the closed orbit solutions within a large momentum range is performed. It starts for a lattice with the initial conditions of the $\beta_{x_{0}}$ and $D_{x_{0}}$ obtained by Eqs. (16) and (17). It is found that stable solutions exist only for a range of $\delta p / p$ in the positive direction from the central momentum up to $+33 \%$. As the values of the initial conditions for $\beta_{x_{0}}$ and $D_{x_{0}}$ are sequentially raised, the stable closed orbit solutions start to show for larger and larger negative range in $\delta p / p$. This procedure is described in Fig. 7. It is important to note that our analysis is done for muon acceleration, with an additional constraint for the path length variation on momentum to be as small as possible. This is optimized when the minimum of the path length occurs in the middle of the momentum range. This condition is not necessary for other applications (such as proton or electron acceleration) and the minimum of the $\langle H\rangle$ function may well come with the reference momentum at the lowest energy. This would allow larger ranges of stable horizontal and vertical betatron tunes. The dependence of the maximum of the orbit offsets on momentum for different values of the initial $\beta_{x_{0}}$ and $D_{x_{0}}$ is presented in Fig. 8. The dependence of the path length variation (units are centimeters) and the maximum orbit offsets on the maximum value of the $H$ function are 


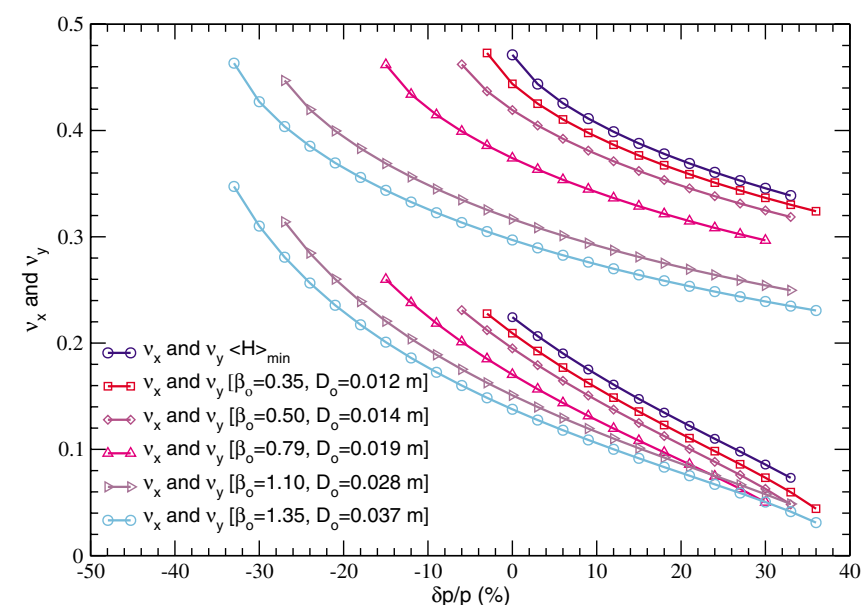

FIG. 7. (Color) Dependence of horizontal and vertical betatron tunes on momentum for various initial conditions for $\beta_{x_{0}}$ and $D_{x_{0}}$. The starting point is the condition for the minimum of the $\langle H\rangle$ function with initial value of the amplitude and dispersion functions $\beta_{0}=0.186 \mathrm{~m} D_{0}=0.008$. Relations between and stable regions for the tunes are shown.

both presented in Fig. 9. The dependence of the normalized dispersion function (amplitude of $\sqrt{H}$ ) on momentum, for our lattice, is presented in Fig. 10. The nonscaling triplet lattice FFAG design has many possible applications. This report concentrates on muon acceleration. Muon acceleration needs to be very fast because of the short muon life time; this imposes special requirements on the lattice design, principally a small variation of the path length. This restriction is not necessary for other applications such as proton acceleration (high intensity proton FFAG, proton therapy), heavy ion acceleration (for carbon therapy or RIA-radioactive ion beams), electron acceleration, etc.

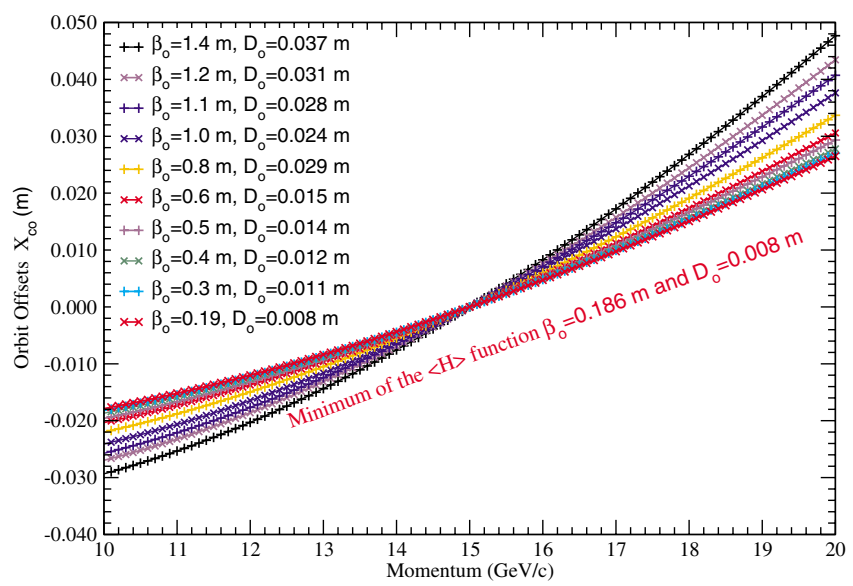

FIG. 8. (Color) Dependence on momentum of maximum orbit offsets. The smallest orbit offsets correspond to the minimum of the $\langle H\rangle$ function. Corresponding initial conditions for the amplitude function $\beta_{x}$ are shown.

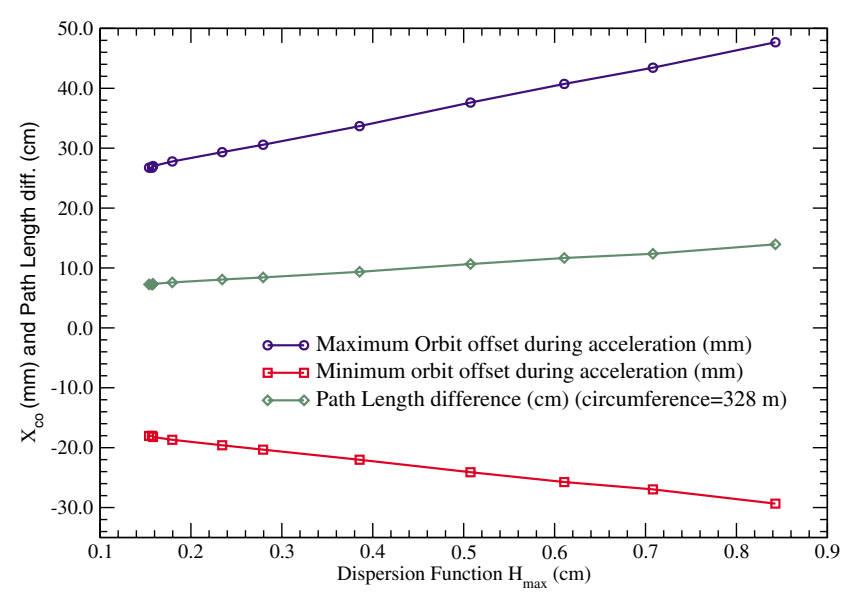

FIG. 9. (Color) Orbit offsets and path length differences vs $H_{\max }$.

There are a few important restrictions on the lattice design for muon acceleration:

\section{B. Small path length difference during acceleration}

The difference in the path length as the energy increases has to be small $(\Delta L \sim 13 \mathrm{~cm})$. The path length dependence on momentum is a parabolic function [24]. It is best to have the same values of the path lengths at the beginning and the end of the acceleration. The path length minimization, for lattices with the same circumference $C_{0}=328$ $\mathrm{m}$, was studied by varying the length of $Q D$, the major bend combined function magnet, between $L_{d}=$ $0.8-1.5 \mathrm{~m}$. The relationship between the path length difference and the magnet length is presented in Fig. 11. A complete optimization has to consider additional parameters such as muon decay time (the longer the circumference the larger muon loss due to the decay), initial emittance of the muon beam to determine more precisely the magnet aperture and cost, number of turns, cavity voltage limita-

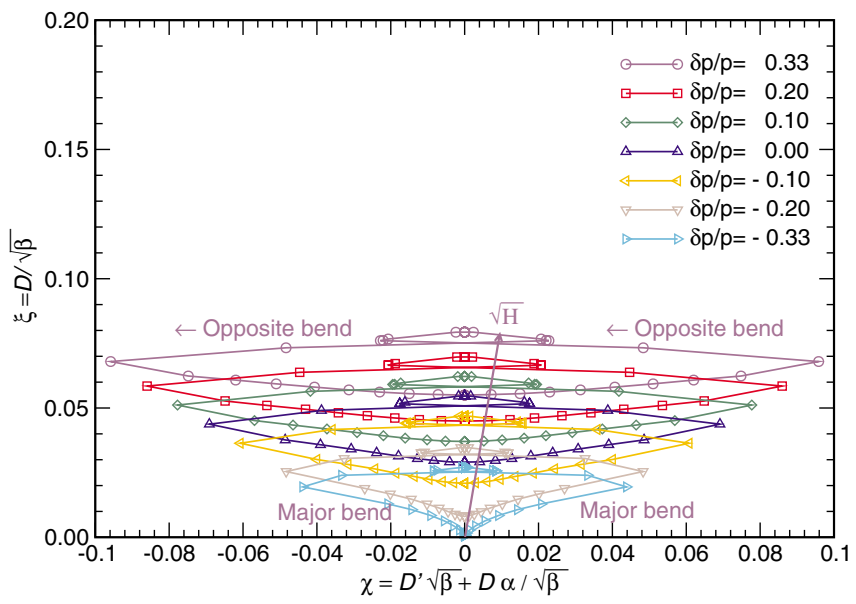

FIG. 10. (Color) Normalized dispersion function during acceleration for different momenta. 


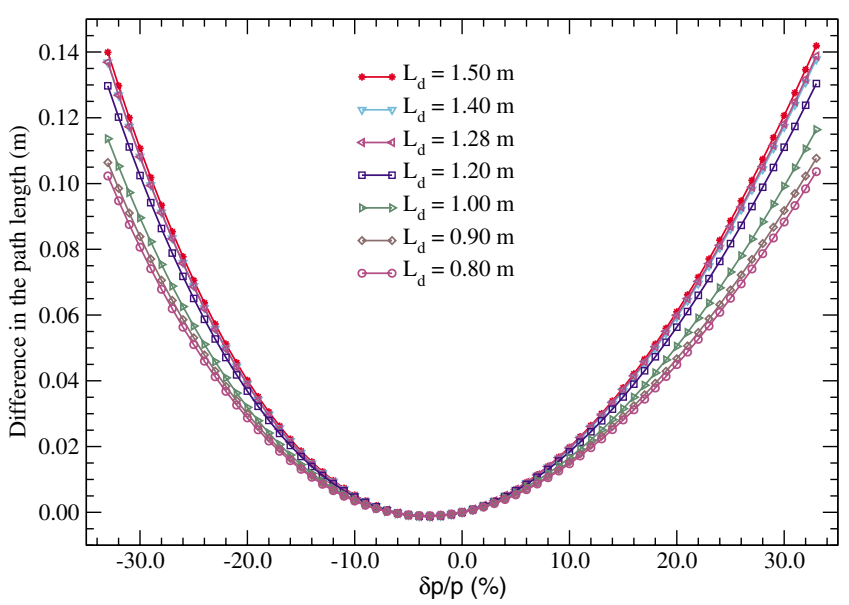

FIG. 11. (Color) Difference in the path length during acceleration. Each lattice has a fixed circumference of $C_{0}=328 \mathrm{~m}$ at central energy; the length of the major combined function magnet varies from $L_{d}=0.8-1.5 \mathrm{~m}$.

tions, beam loading, etc. More detailed studies considering cost optimization have been performed elsewhere [30]. It is clear that larger circumference reduces the strength of the magnetic field, allows more room for the number of cavities, but reduces the number of available muons because of their decay. On the other hand a larger circumference reduces the path length difference and allows better conditions in the longitudinal motion as explained in the Sec. IV. The magnetic fields and gradients in lattices with the circumference $C_{0}$ fixed at $328 \mathrm{~m}$, as presented in Fig. 11, are shown in detail in Table II. Analytical calculations [24] of path length, with an approximation where the higher order terms in $A_{f} / \rho_{f 0}$ and $A_{d} / \rho_{d 0}$ were neglected $\left(A_{f}\right.$ and $A_{d}$ are the amplitudes of the betatron motion), have shown very good agreement with the exact solutions by PTC and the COSY INFINITY results with 8th order in momentum.

\section{Momentum compaction $\boldsymbol{\alpha}_{\boldsymbol{c}}$}

The momentum compaction is defined as [29]

$$
\alpha_{c} \equiv \frac{1}{C} \frac{d \Delta C}{d \delta}=\frac{1}{C} \oint \frac{D(s)}{\rho} d s .
$$

TABLE II. Major magnet length, number of periods, magnetic fields, and gradients in the $Q F$ and $Q D$ magnets.

\begin{tabular}{lccccc}
\hline \hline$L_{d}(\mathrm{~m})$ & Periods & $B_{f}(\mathrm{~T})$ & $B_{d}(\mathrm{~T})$ & $G_{d}(\mathrm{~T} / \mathrm{m})$ & $G_{f}(\mathrm{~T} / \mathrm{m})$ \\
\hline 0.80 & 75 & -1.70 & 7.704 & -70.83 & 63.16 \\
0.90 & 74 & -1.95 & 7.233 & -65.66 & 65.38 \\
1.00 & 72 & -2.00 & 6.686 & -56.65 & 62.43 \\
1.20 & 68 & -2.00 & 5.852 & -44.09 & 56.68 \\
1.28 & 67 & -2.15 & 5.614 & -40.28 & 56.43 \\
1.40 & 66 & -2.20 & 5.225 & -36.77 & 56.63 \\
1.50 & 66 & -2.29 & 4.946 & -34.27 & 57.19 \\
\hline \hline
\end{tabular}

With the approximately parabolic dependence of path length on momentum the minimum value occurs near the central energy. The dependence of momentum compaction on energy during acceleration is to the first order a linear function as shown in Fig. 12. The zero value of $\alpha_{c}$ occurs near, but not exactly at, the reference momentum of $p_{0}=$ $15 \mathrm{GeV} / c$. This is a result of the optimization for the path length dependence.

\section{Orbit offsets during acceleration}

The emittance of the incoming muon beam to be accelerated by the nonscaling FFAG depends on the efficiency and properties of the previous stages of acceleration and cooling. The needed magnet size is mostly determined by the beam size, but it is also important to reduce the beam offsets during acceleration. The major advantage of the nonscaling FFAG design with respect to scaling version is the dramatically smaller orbit offset during acceleration. The dependence of orbit offset on the maximum value of the $H$ function is shown in Fig. 8. The small orbit offset during acceleration as shown by Fig. 13 represents the major advantage of the nonscaling design compared to the scaling one. Orbits at different momenta are parallel to each other within the drift between the two focusing combined function magnets, but the orbits are not parallel to each other in the rest of the cell due to the strong focusing and magnet edge effects.

\section{E. Small circumference}

The smaller the circumference and orbit offsets, the lower is the cost for the whole machine. The scaling FFAG design requires longer negative bend magnets: In [2], the relationship between the negative vs positive bend is $\theta_{2} / \theta_{1}=2 / 3$. This ratio means that the total magnet length needed, in this case, is $(1.5+1) /(1.5-1)=5$ times that needed just for bending, so the circumference has to be large. The negative bend also affects the disper-

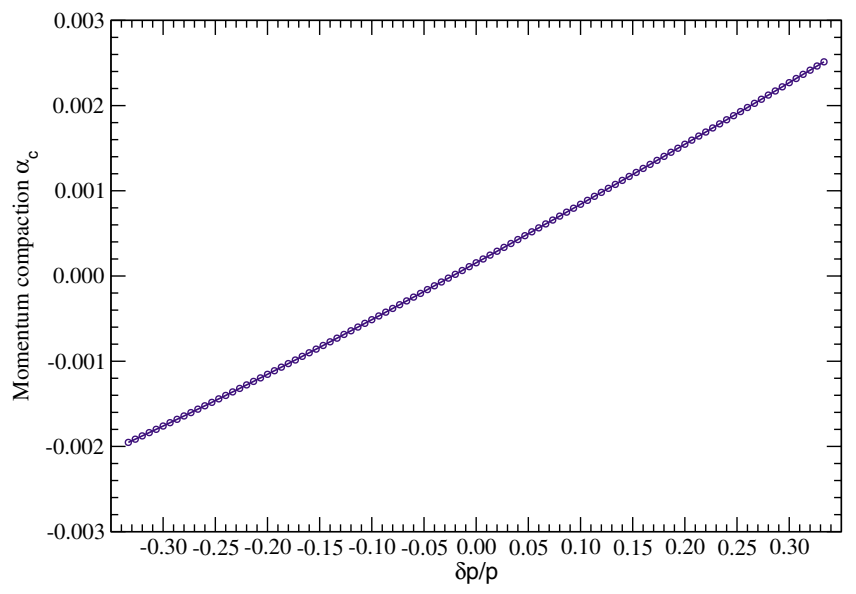

FIG. 12. (Color) Dependence of momentum compaction $\alpha$ on momentum 


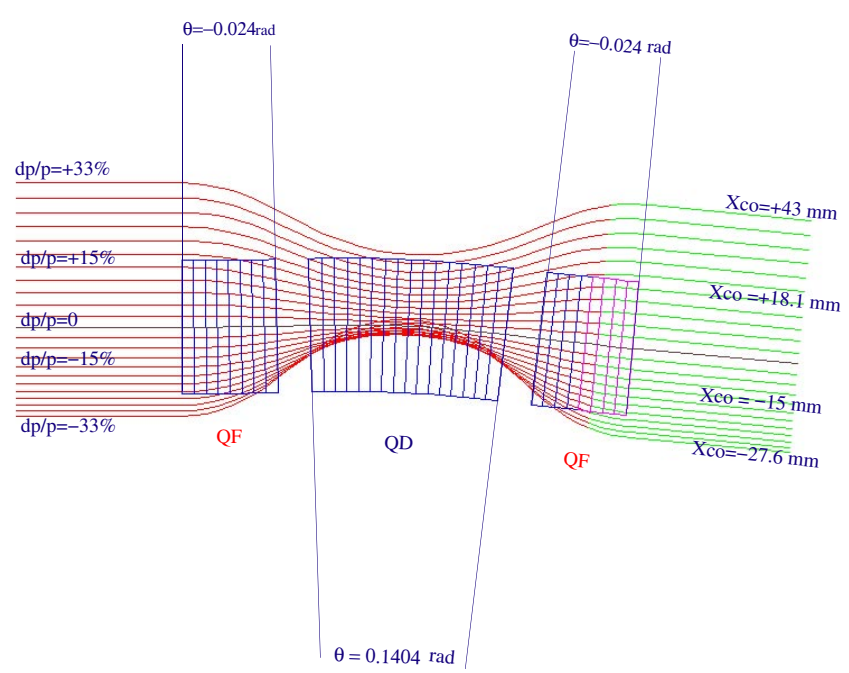

FIG. 13. (Color) Orbit offsets obtained by the polymorphic tracking code in one of the FFAG examples.

sion function, as defined in Eq. (18). The value of the momentum compaction $\alpha_{c}$ is affected by the size of the negative bend. This is due to its negative sign in the integral of the dispersion function. Equal values of the path lengths at the lowest and highest momentum in the parabola, as shown in Fig. 11, are obtained by varying the ratio between the opposite and major bend $\theta_{2} / \theta_{1}$. The parabola optimization is presented in Fig. 14. Equal path lengths at the beginning and end of the parabola are obtained for the ratio between opposite and major bend as $\theta_{Q F} / \theta_{Q D}=0.184$.

\section{A. Amplitude functions dependence on momentum}

The dependence on momentum of the horizontal and vertical amplitude functions $\sqrt{\beta_{x}}$ and $\sqrt{\beta_{y}}$ and of the dispersion function $D_{x}$ are shown in Figs. 15-17.

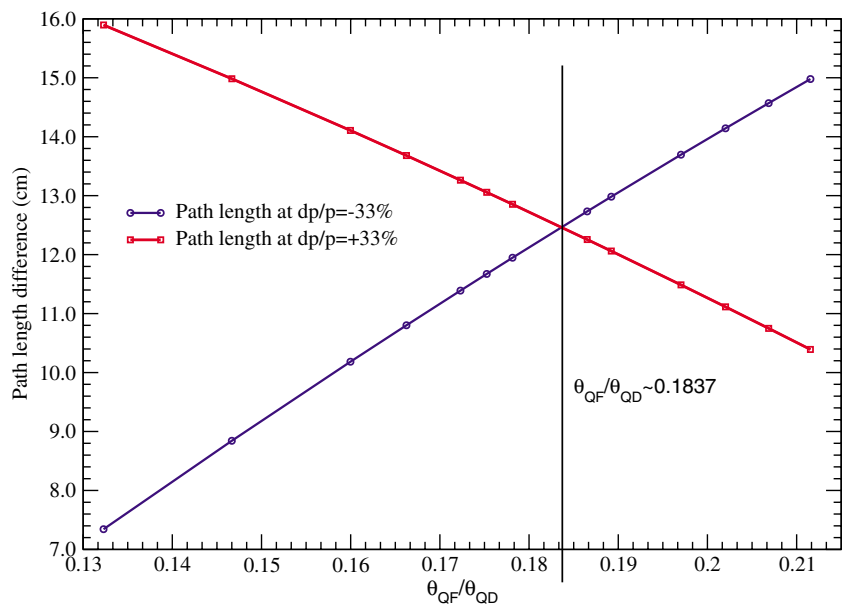

FIG. 14. (Color) Opposite bend vs major bend for equalizing the parabola.

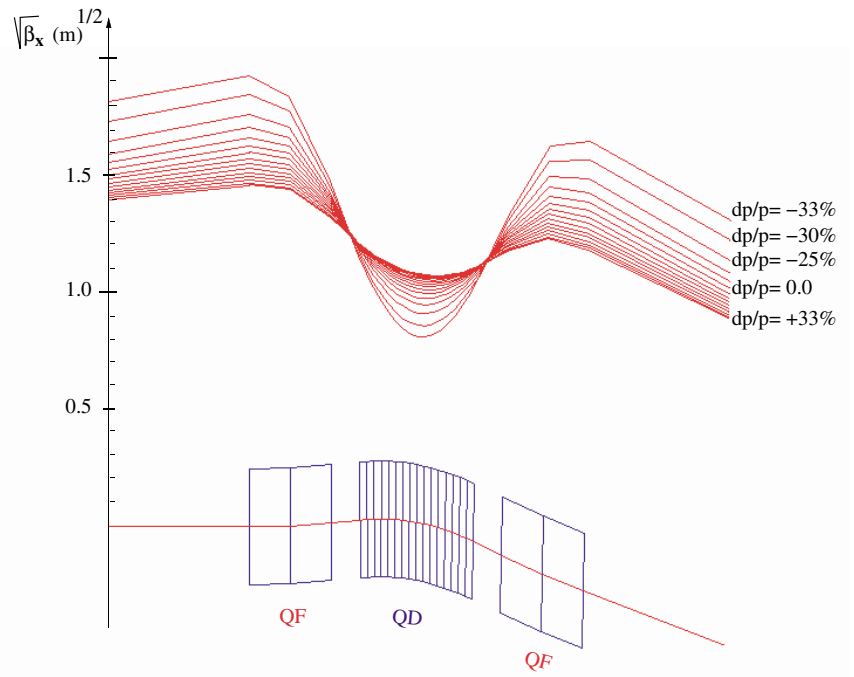

FIG. 15. (Color) Dependence on momentum of $\sqrt{\beta_{x}}$ as calculated by the PTC code.

\section{G. Transverse tracking}

The nonscaling FFAG presented here does not include any nonlinear fields. Particle tracking in the transverse plane at the muon central energy of $15 \mathrm{GeV}$ was performed for 1500 turns, with different initial conditions of the horizontal and vertical positions and slopes. A stable motion in the horizontal phase space is shown in Fig. 18.

A study of the particle motion during acceleration is shown in the next section, while particle tracking during acceleration including effects of magnet misalignment and gradient errors have been studied elsewhere [31,32].

\section{ACCELERATION}

Muons require a short acceleration cycle. For reasonable amplifier power this precludes variation of the rf parame-

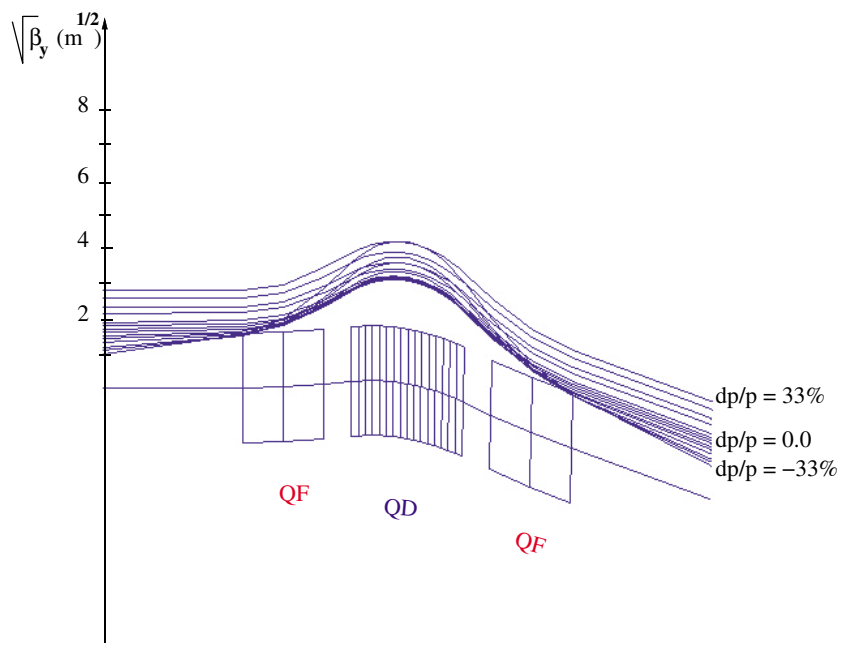

FIG. 16. (Color) Dependence on momentum of $\sqrt{\beta_{y}}$ as calculated by the PTC code. 


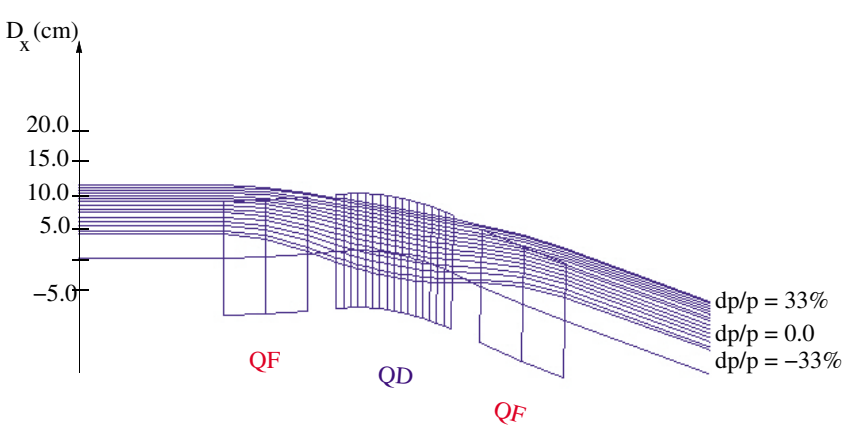

FIG. 17. (Color) Dependence of horizontal dispersion $D_{x}$ on momentum as calculated by the PTC code.

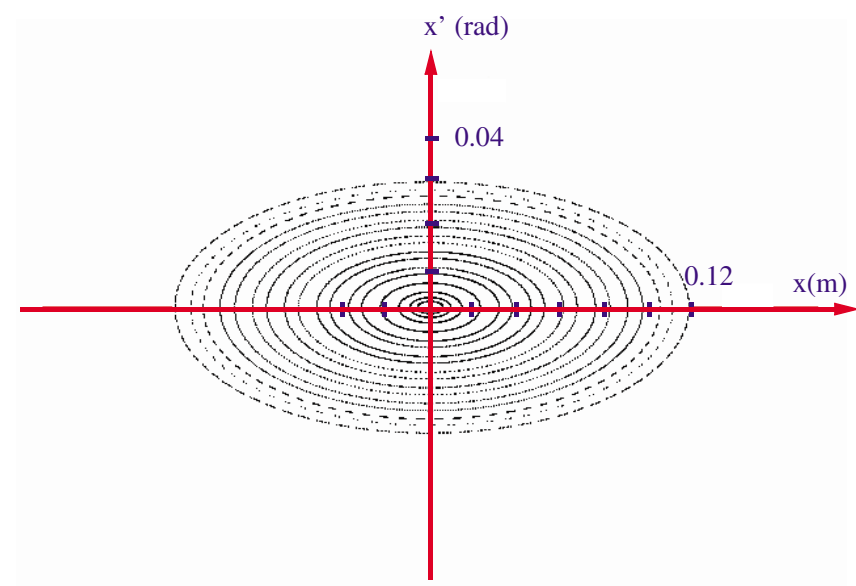

FIG. 18. (Color) Results from transverse particle tracking at the central $15 \mathrm{GeV}$ muon energy. This is a result obtained by the COSY INFINITY code.

ters during the cycle [13]. In particular, all the energy which will accelerate the muons must reside in the cavity electromagnetic field at the time of injection. We will consider standing wave superconducting cavities, similar to the Cornell/CERN design [15]. We take a maximum accelerating voltage per cavity of $V=12 \mathrm{MV}$ with a stored energy of $U=955 \mathrm{~J}$. The stored energy and voltage are related via

$$
U=\frac{V^{2}}{2 \omega_{r f} R / Q},
$$

where $\omega_{r f} / 2 \pi=f_{r f} \approx 200 \mathrm{MHz}$, and we use the circuit definition with $R / Q=60 \Omega$. To simulate the system we lump the $N$ rf cavities into a single thin lens. Let $\tau_{n}$ be the time a particle arrives at this lens on turn $n$, and let $E_{n}$ be the energy a particle has after traversing the cavity. The update equations are then

$$
\begin{gathered}
\tau_{n+1}=\tau_{n}+T\left(E_{n}\right), \\
E_{n+1}=E_{n}+q N V\left(\tau_{n+1}\right),
\end{gathered}
$$

where $V(t)$ is the voltage on a single cavity as a function of time, $q$ is the charge per particle, and $T(E)$ is the revolution period as a function of energy. To get the equation for $V(t)$ we model a cavity as a parallel $L C$ resonator. The current through the capacitor is $I_{C}=C d V / d t$ and the current through the inductor is $I_{L}=\int d t V / L$. The total current driving the cavity is then $I_{L}+I_{C}$. The net current is the sum of the contributions from the power amplifier and the beam. Since the fill time of the cavity is long compared with the acceleration time we take the voltage due the amplifier to be $V_{a}=\hat{V} \sin \left(\omega_{r f} t+\phi\right)$ where $\hat{V}$ and $\phi$ are constants. The voltage due to the beam current, $V_{b}(t)$ is identically zero before the beam is injected at $t=0$. The beam image currents on the cavity walls charge the capacitive gap so the negative of the beam current generates the beam induced voltage,

$$
-\left(\frac{R}{Q}\right) I_{b}(t)=\frac{1}{\omega_{r f}} \frac{d V_{b}}{d t}+\omega_{r f} \int_{0}^{t} V_{b}\left(t_{1}\right) d t_{1},
$$

where $I_{b}(t)$ is the beam current as a function of time at the rf cavity, and $R / Q=\omega_{r f} L=1 / \omega_{r f} C$. The total voltage is then given by $N V=N\left(V_{a}+V_{b}\right)$. We consider the three cases shown in Fig. 19. The lattice and rf parameters are given in Table III.

Figures 20-22 show the initial and final rf voltage and the bunch evolution for a total beam charge of $2.5 \mu \mathrm{C}$ for the three cases in Table III. For all cases the initial emittance was $0.2 \mathrm{eV}-\mathrm{s}$ per bunch with an initial energy spread of $\pm 400 \mathrm{MeV}$. For a lattice with the $L_{d}=0.8 \mathrm{~m}$ of the major bend, $40 \%$ of the energy stored in the cavities is transmitted to the beam. For $L_{d}=1.5 \mathrm{~m}$ lattice this fraction is $32 \%$. However, the $960 \mathrm{MV}$ required for $L_{d}=$ $1.5 \mathrm{~m}$ requires more Cornell cavities than lattice periods, requiring the technology improvements.

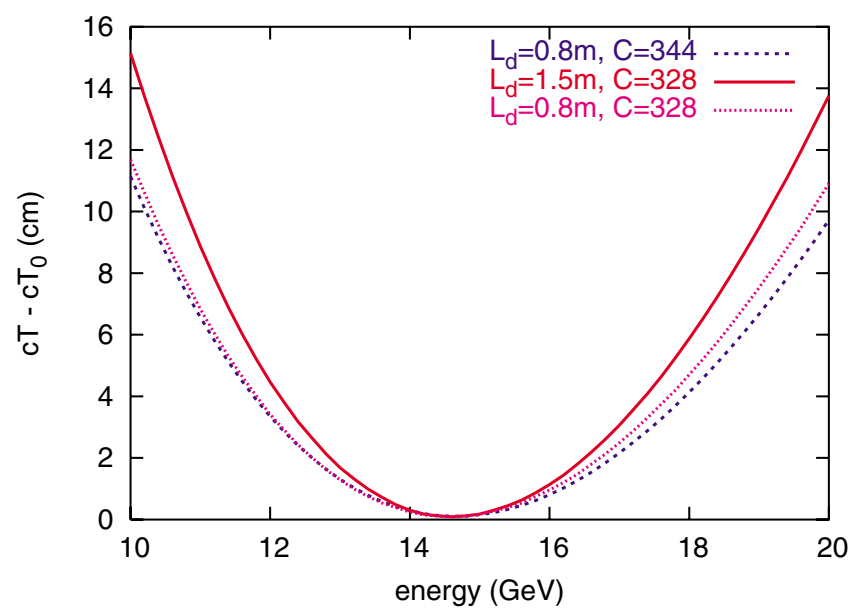

FIG. 19. (Color) Variation in revolution period with energy for three lattices. Parameters are given in Table III. 
TABLE III. Parameters for the rf simulations.

\begin{tabular}{lccc}
\hline \hline \multicolumn{1}{c}{ Parameter } & & & \\
\hline$L_{d}(\mathrm{~m})$ & 0.8 & 1.5 & 0.8 \\
Circumference $(\mathrm{m})$ & 344 & 328 & 328 \\
Harmonic number & 229 & 220 & 220 \\
$f_{r f} \mathrm{MHz}$ & 199.56 & 201.07 & 201.07 \\
Number of Cornell cavities & 60 & 80 & 60 \\
Voltage per turn (MV) & 720 & 960 & 720 \\
Number of turns & 16 & 12 & 16 \\
Lattice periods & 80 & 66 & 75 \\
\hline \hline
\end{tabular}

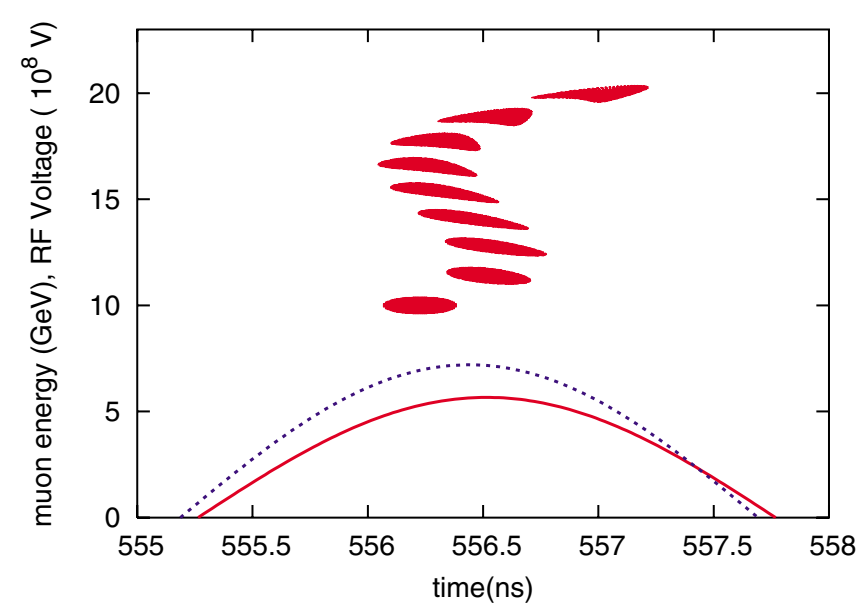

FIG. 20. (Color) Initial (dash) and final (full) rf voltage, and evolution of the bunch on turns $0,2, \ldots 16$, for $L_{d}=0.8 \mathrm{~m}$ and $C_{0}=344 \mathrm{~m}$.

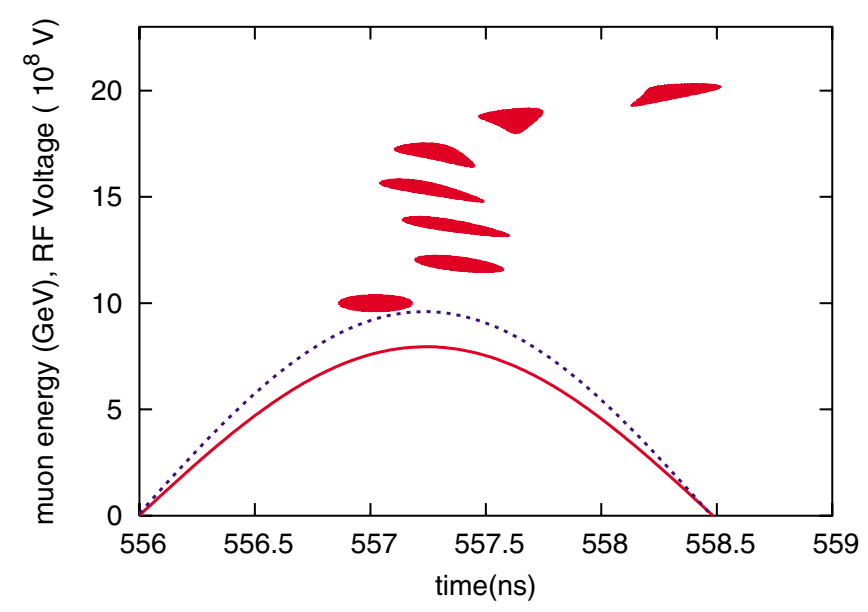

FIG. 21. (Color) Initial (dash) and final (full) rf voltage, and evolution of the bunch on turns $0,2, \ldots 12$, for $L_{d}=1.5 \mathrm{~m}$ and $C_{0}=328 \mathrm{~m}$.

\section{CONCLUSIONS}

We have presented an alternative "nonscaling triplet design" of the fixed field alternating gradient accelerator. Some disadvantages like the large momentum offsets,

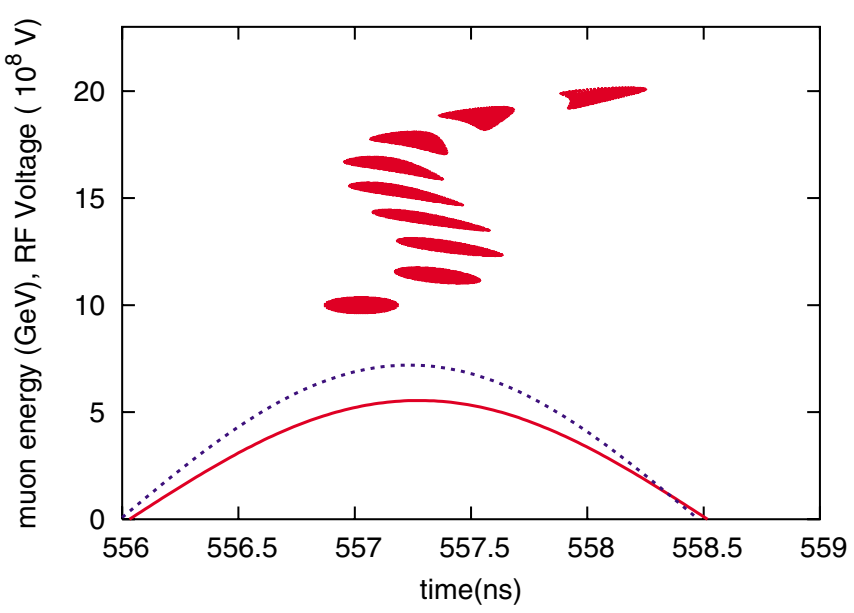

FIG. 22. (Color) Initial (dash) and final (full) rf voltage, and evolution of the bunch on turns $0,2, \ldots 16$, for $L_{d}=0.8 \mathrm{~m}$ and $C_{0}=328 \mathrm{~m}$.

large circumference, large magnets sizes of the standard [2] scaling FFAG are improved or eliminated. The acceleration of muons for the "Neutrino Factory Project" or the "Muon Collider Project" has to be in a very short time due to a short muon lifetime. We have shown a lattice design for muon acceleration from 10 to $20 \mathrm{GeV}$. The properties of the lattice and magnet elements are shown at the reference momentum and through the acceleration range. The best efficiency with respect to particle stability, cost of the rf cavities and magnets is obtained when the number of muon bunch passages during acceleration is less than 15 . This conclusion comes from the acceleration simulations where significant bunch distortions occur at the last few turns, especially if a larger number of turns is applied (part of it is shown in Figs. 20-22, in the bunch shape during the last few turns). The maximum orbit offsets during acceleration are within $-30<x<48 \mathrm{~mm}$. This reduces the aperture and the magnet size compared to the scaling design. We have studied and compared other possible applications like proton [33] and electron acceleration [34]. The proton and electron designs have no restrictions imposed by the muon lifetime and have shown many advantages with respect to other ways of accelerations such as multiturn through the linear accelerator with many arcs for each energy, or ramping magnetic field in synchrotrons. Other examples of muon [35], proton, and electron acceleration for the proof of principle $10 \mathrm{MeV}$ electrons and for electron acceleration up to $10 \mathrm{GeV}$ for e-RHIC (future proposal for the electron-proton or heavy ion Relativistic Heavy Ion Collider) are shown in other publications [34].

\section{ACKNOWLEDGMENTS}

We thank Robert Palmer, J. Scott Berg, and Etienne Forest for rewriting the FFAG lattice of our example for PTC, as well as S. Koscielniak for insightful comments. This manuscript has been authored by Brookhaven Science 
Associates, LLC under Contract No. DE-AC02-98CH1886 with the U.S. Department of Energy.

[1] T. Ohkawa, University of Tokyo, Tokyo, Japan, FFAG structure suggested earlier at a Symposium on Nuclear Physics of the Physical Society of Japan in 1953 (private communication).

[2] K. R. Symon, D. W. Kerst, L. W. Jones, L. J. Laslett, and K. M. Terwilliger, Phys. Rev. 103, 1837 (1956).

[3] A. Kolomensky et al., Zh. Eksp. Teor. Fiz. 33, 298 (1957).

[4] M. Yoshimoto et al., in Proceedings of the 8th European Particle Accelerator Conference, Paris, France, 2002 (EPS-IGA and CERN, Geneva, 2002), pp. 1319-1322.

[5] M. Aiba et al., in Proceedings of the 8th European Particle Accelerator Conference, Paris, France, 2002 (Ref. [4]), pp. 1027-1030.

[6] S. Machida, in Proceedings of FFAG 03, KEK, Japan, 2003, http://hadron.kek.jp/FFAG/FFAG03_HP/index.html

[7] D. Trbojevic, in Proceedings of FFAG Workshop, TRIUMF, Vancouver B.C., 2004, http://www.triumf.ca/ ffag2004/.

[8] Lee C. Teng, Rev. Sci. Instrum. 27, 1051 (1956).

[9] M. Craddock, CERN Courier 44, 23 (2004); http:// www.cerncourier.com/main/article/44/6/17.

[10] E. Keil and A. M. Sessler, Report No. CERN-AB-2004033(ABP) [Nucl. Instrum. Methods Phys. Res., Sect. A (to be published)].

[11] M. M. Alsharo'a et al., Phys. Rev. ST Accel. Beams 6, 081001 (2003).

[12] C. Johnstone, Nucl. Instrum. Methods Phys. Res., Sect. A, 503, 301 (2003).

[13] C. Johnstone and S. Koscielniak, Nucl. Instrum. Methods Phys. Res., Sect. A 503, 445 (2003).

[14] S. Koscielniak and C. Johnstone, Nucl. Instrum. Methods Phys. Res., Sect. A 523, 25 (2004).

[15] R. L. Geng, P. Barnes, D. Hartill, H. Padamsee, J. Sears, S. Calatroni, E. Chiaveri, R. Losito, and H. Preis, in Proceedings of the Particle Accelerator Conference, Portland, Oregon, 2003 (IEEE, Piscataway, NJ, 2003), pp. 1309.

[16] E. D. Courant, S. J. Berg, D. Trbojevic, R. Talman, and A. A. Garren, in Proceedings of the Particle Accelerator Conference, Portland, Oregon, 2003 (Ref. [15]), IEEE Catalog No. 03CH37423, pp. 3485-3487.

[17] A. A. Garren, A. S. Kenney, E. D. Courant, A. D. Russell, and M.J. Syphers, A User's Guide to SYNCH, No. FERMILAB-FN-0420, 1985, p. 151.

[18] E. Forest, Frank Schmidt, and Eric McIntosh, Introduction to the Polymorphic Tracking Code, http://frs.home.cern.ch/frs/Source/MAD-X_Day/PTC/etienne_ptc.pps.
[19] M. Berz and J. Hoefkens, COSY INFINITY Version 8.1 Programming Manual No. MSUHEP-20704 (Department of Physics and Astronomy, Michigan State University, East Lansing, MI, 2002), http://cosy.pa.msu.edu/cosymanu/index.html.

[20] F. Méot and S. Valero, ZGOUBI User's Guide, Version 4.3, CEA DSM DAPNIA-02-395 (Saclay, F91191 Gif-surYvette Cedex, France, 2002).

[21] F. Méot, in Proceedings of the KEK FFAG Workshop, Tsukuba, Japan, 2003 (Ref. [6]).

[22] D. L. Bruhwiler, J.R. Gary, and S.G. Shasharina, in Proceedings of the Particle Accelerator Conference, Lucerne, 2004 (EPS-AG, Lucerne, 2004), pp. 1171-1173.

[23] E. Forest, M. Reusch, D. L. Bruhwiler, and A. Amiry, Part. Accel. 45, 65 (1994).

[24] M. Craddock, in Proceedings of FFAG Workshop, TRIUMF, Vancouver B.C., 2004 (Ref. [7]).

[25] D. Kaltchev, in Proceedings of FFAG Workshop, TRIUMF, Vancouver B.C., 2004 (Ref. [7]).

[26] S. Koscielniak, in Proceedings of FFAG Workshop, TRIUMF, Vancouver B.C., 2004 (Ref. [7]).

[27] A. G. Ruggiero, Brookhaven National Laboratory Internal Note No. C-A/AP/148, 2004.

[28] D. Trbojevic, D. Finley, R. Gerig, and S. Holmes, in Proceedings of Second European Particle Accelerator Conference, Nice, 1990, edited by P. Martin and P. Mandrillon (Editions Frontières, Gif-sur-Yvette, France, 1990), pp. 1536-1538.

[29] S. Y. Lee, Accelerator Physics (World Scientific Publishing Co., Pte. Ltd., Singapore,1999), p. 454.

[30] J.S. Berg, in Proceedings of the 17th International Conference on Cyclotrons and Their Applications, Tokyo, 2004, http://ribfweb1.riken.go.jp/cyc2004/presentation/19C2_J_Berg.pdf

[31] S. Machida, in Proceedings of the FFAG workshop in Fermilab, 2005, http://www.bt.pa.msu.edu/ffag/ agenda.html.

[32] E. Keil and A. Sessler, in Proceedings of FFAG Workshop, TRIUMF, Vancouver B.C., 2004 (Ref. [7]). http:// www.triumf.ca/ffag2004/talks/EberhardKeil_doublet.pdf.

[33] D. Trbojevic, A. G. Ruggiero, E. Keil, N. Neskovic, and A. Sessler, in Proceedings of the 17th International Conference on Cyclotrons and Their Applications, Tokyo, 2004 (Ref. [30]).

[34] D. Trbojevic, M. Blaskiewicz, E. D. Courant, A. Ruggiero, J. Kewisch, T. Roser, and N. Tsoupas, in Proceedings of the Particle Accelerator Conference, Lucerne, 2004 (Ref. [22]).

[35] D. Trbojevic, J. S. Berg, M. Blaskiewicz, E. D. Courant, R. Palmer, and A. A. Garren, in Proceedings of the Particle Accelerator Conference, Portland, Oregon, 2003 (Ref. [15]), pp. 1816-1819. 